\section{Premise to implement a grading system to evaluate the sanitary level in food service establi- shments in Milan, Italy}

\author{
Katia Razzini, ${ }^{1}$ Claudia Maria Balzaretti ${ }^{2}$ \\ 1U.N.P.I.S.I., Perugia, Italy \\ ${ }^{2}$ Department of Veterinary Science for \\ Health, Animal Production and Food \\ Production, University of Milan, Italy
}

\begin{abstract}
The regulatory framework of the official controls on food safety, the criteria and methods from the planning of interventions in the field of official control to the management of information flows, and the standards described in the operation manual of the local competent authorities drafted by the Lombardy Region (2011) were evaluated. A questionnaire consisting of $n .10$ questions with multiple answers draft in partnership with EPAM (the Association of Provincial Public Retail and catering businesses in Milan) to n. 107 Food service establishments of Milan shows that $92 \%$ of managers approve the introduction of a grading system. The regulatory framework is planned to support the implementation of risk assignment, unfortunately the attribution of risk category of retail and catering businesses is still different among regions.
\end{abstract}

\section{Introduzione}

Con il Libro Bianco sulla sicurezza alimentare (Commissione Europea, 2000b) si evidenzia come nel caso di strutture e servizi di ristorazione, seppur indicato chiaramente, non sempre il flusso d'informazioni con il consumatore è sufficiente a soddisfare la necessità d'informazione e, non sempre il consumatore può conoscere il livello igienico sanitario del servizio offerto in modo trasparente e facilmente leggibile.

Il concetto di $A B C$ grading system concernente l'igiene pubblica nasce negli USA nel 1920 quando l'U.S. Public Health Service (USPHS) creò il primo codice ad applicazione volontaria, denominato Model Milk Code. Il codice aveva lo scopo di fornire indicazioni sulla sicurezza dei processi di produzione e di vendita del latte, comunicandone il grado tramite una lettera da applicare direttamente sulle bottiglie (McDean, 1997). Il modello creato nel 1920 influenza positivamente la sicurezza alimentare americana e nel 1932, sulla base dei Milk Code, nasce il primo Model Food Code per poi prendere corpo così come lo conosciamo nei primi anni del 1960. La maggior parte delle norme che regolavano l'invio dei punteggi e dei voti furono lasciate ai singoli stati e alle loro amministrazioni locali. In particolare Tennessee e North Carolina furono i primi ad applicare una legislazione obbligatoria che prevedeva tale codice.

Solo negli ultimi anni il Grade Card System ha preso dignità a livello internazionale, come evidenzia uno studio di fattibilità condotto nel luglio del 2000 dall'Ottawa-Carleton Health Department in Canada (Boehnke, 2000). Tale rapporto mostra come su settantanove giurisdizioni responsabili dell'ispezione di ristoranti, tra cui paesi appartenenti alla Comunità Europea, all'America meridionale e all'Australia, solamente gli Stati Uniti d'America e la Repubblica di Singapore adottavano un sistema di valutazione igienico-sanitaria a punti, e solo in seguito a tale studio lo stesso Canada decise di introdurre tale sistema di valutazione. A oggi numerosi paesi applicano, anche in maniera volontaria, sistemi di valutazione riconducibili a quello americano, in particolare la Nuova Zelanda, e, in Europa: Danimarca, Belgio, Regno unito e Scozia.

La crescente richiesta d'informazioni da parte dei consumatori ha portato alla formazione di banche dati accessibili tramite il web, nelle quali sono registrati i punteggi di tutti i ristoranti e i risultati di tutte le ispezioni precedenti. Il web ha reso ancora più fruibile questo servizio mettendo a disposizione del consumatore informazioni di rapida consultazione. Gli obblighi imposti all'Autorità Competente dalla Comunità Europea riguardano in particolare: le modalità di programmazione dell'attività di vigilanza e controllo basate sempre più sulla definizione del livello di rischio applicato non solo alle singole categorie, ma anche alle singole imprese, secondo quanto emerge dalla storia di ognuna di esse, attraverso l'opportuna implementazione dell'anagrafe delle aziende. La Circolare n.6/SAN/2007 sulla Programmazione degli interventi in materia di controllo ufficiale dei prodotti alimentari (Regione Lombardia, 2007b), è il documento dove per la prima volta è stato introdotto il concetto di graduazione degli interventi di vigilanza in funzione della caratterizzazione del rischio delle industrie alimentari.

Le attività di fatto erano ancora ripartite sulla base del Modello A allegato al D.P.R. 14/7/1995 (Repubblica Italiana, 1995), mantenendo una suddivisione in raggruppamenti e categorie. I dati relativi alla ristorazione pubblica sono che la frequenza di ispezione per ristoranti, trattorie, pizzerie, tavole calde è di ogni 3-5 anni, aziende agrituristiche con somministrazione è di 1-2 anni, per fiere, feste popolari a carattere temporaneo sono il $10 \%$ all'anno, mentre bar e le tavole fredde ogni 4-6
Correspondence: Claudia Maria Balzaretti, Department of Veterinary Science for Health, Animal Production and Food Production, University of Milan, Via Celoria 10, 20133 Milan, Italy.

Tel./Fax: +39.02 .503111$

E-mail: claudia.balzaretti@unimi.it

Key words: Grading system; Food service establishment; Consumers.

Received for publication: 8 July 2014 . Accepted for publication: 7 January 2015.

This work is licensed under a Creative Commons Attribution 3.0 License (by-nc 3.0).

(O) Copyright K. Razzini et al., 2015

Licensee PAGEPress, Italy

Italian Journal of Food Safety 2015; 4:4514

doi:10.4081/ijfs.2015.4514

anni. La Circolare n.32/SAN/2007 (Regione Lombardia, 2007a) sulla predisposizione e presentazione dei Piani integrati delle attività di prevenzione e dei controlli prevede che le singole ASL predispongano una: i) suddivisione delle attività soggette a controllo in macro categorie omogenee; ii) attribuzione di un livello di rischio alle varie categorie; iii) Effettuazione di una categorizzazione del rischio per singola unità soggetta a controllo all'interno delle macro categorie.

Scopo di questo lavoro è stato quello di acquisire informazioni sullo stato attuale della regolamentazione per poter formulare un protocollo sperimentale per l'applicazione anche in Italia di un sistema grading system fruibile anche dal consumatore finale.

\section{Materiali e Metodi}

Sono stati raccolti dalle 15 ASL in Regione Lombardia i dati relativi all'anno 2010 e inerenti il numero dei Pubblici Esercizi, delle attività di ristorazione e i codici ATECO sez. $554 \mathrm{e}$ 553. È stato valutato il livello di rischio attribuito dalla Regione, il livello di rischio assegnato dalle rispettive ASL, il numero di controlli e il livello di copertura.

È stato quindi realizzato un questionario (Tabella 1) in collaborazione con EPAM (Associazione Provinciale Milanese dei Pubblici Esercizi) composto da 10 domande a risposta chiusa, somministrato a 107 responsabili di Pubblici Esercizi della città di Milano. È stata valutata la normativa a disposizione dell'Autorità Sanitaria in Regione Lombardia considerando in particolare gli obblighi imposti dalla Comunità Europea che riguardano: 1Le modalità di programmazione dell'attività di vigilanza e controllo basate sempre più sulla 
definizione del livello di rischio applicato non solo alle singole categorie, ma anche alle singole imprese, secondo quanto emerge dalla storia di ognuna di esse, attraverso l'opportuna implementazione dell'anagrafe delle aziende; 2-Le modalità di svolgimento dell'attività ispettiva che integra l'attività di vigilanza tradizionale, con l'attività di audit, per la quale sono state recentemente fornite le indicazioni operative; 3 - Le modalità di gestione dei dati derivanti dall'attività di vigilanza e controllo nei confronti delle singole imprese, attraverso l'implementazione di un sistema informativo riportante l'anagrafica, l'attività svolta e gli esiti. Si è quindi verificata l'esistenza di adeguate definizioni dei criteri di valutazione quale condizione necessaria alla realizzazione di un Modello di classificazione delle attività della Ristorazione Pubblica (tipo grading system) per garantire quei principi di omogenea valutazione ed equivalenza dei controlli ufficiali, all'interno di tutto il territorio Regionale. È stata inoltre verificata l'interferenza normativa esistente in materia di violazione della Privacy, di tutela dell'iniziativa economica privata e del diritto alla difesa dell'esercente inerente alle attività del settore alimentare.

\section{Risultati e Discussione}

L'analisi della documentazione fornita dalle ASL (Tabelle 2 e 3) ha evidenziato una disparità di attribuzione del livello di rischio tra $\mathrm{i}$ diversi Dipartimenti di Prevenzione. Dall'analisi dei valori assegnati dalla Regione esiste una sostanziale conformità, che induce l'osservazione che l'attività di valutazione del rischio è ancora ripartita più con la logica delle categorie/raggruppamenti di appartenenza che su una effettuazione di una categorizzazione del rischio per singola unità soggetta a controllo all'interno delle macro-categorie. Da questa valutazione si determina quindi l'indicazione del livello di copertura, che dipende dalle risorse disponibili e dalla realtà territoriale estremamente variabile.

In particolare, per quanto riguarda i bar dalla Tabella 2 emerge che il livello di copertura medio è circa il $17 \%$ con variazioni comprese tra un minimo del $6 \%$ e un massimo del $32 \%$, che corrispondono ad una frequenza di ispezione variabile tra 1 e 20 anni con un valore medio che si attesta intorno ai 5 anni. Per quanto riguarda invece i ristoranti, dalla Tabella 3 si evince che il livello di copertura medio è pari al $22 \%$ (minimo $6 \%$ e massimo $50 \%$ ), pari ad una frequenza di ispezione media di circa 5 anni (da 1 volta ogni 20 anni a 1 volta ogni 2 anni).

Si nota anche come nell'ASL identificata con

Tabella 1. Esempio di questionario somministrato agli esercenti dei pubblici esercizi.

\begin{tabular}{|c|c|c|c|c|c|c|}
\hline & Domanda & & & Risposte & & \\
\hline 1 & $\begin{array}{l}\text { Come giudica questa } \\
\text { iniziativa in termini } \\
\text { di ricaduta sul settore? }\end{array}$ & Un danno assoluto & $\begin{array}{l}\text { Una opportunità } \\
\text { e incentivo } \\
\text { alla qualificazione }\end{array}$ & $\begin{array}{l}\text { Un sistema } \\
\text { premiante } \\
\text { chi opera secondo } \\
\text { criteri di qualità }\end{array}$ & $\begin{array}{l}\text { Una eventualità } \\
\text { perauspicabile } \\
\text { ma di difficile } \\
\text { realizzazione }\end{array}$ & Non risponde \\
\hline 2 & $\begin{array}{l}\text { Nell'eventualità lei giudichi } \\
\text { non positivamente } \\
\text { o non fattibile } \\
\text { l'iniziativa cosa le suscita } \\
\text { maggiori preoccupazioni? }\end{array}$ & $\begin{array}{l}\text { Non omogeneità } \\
\text { di controllo } \\
\text { da parte dell'A.S. }\end{array}$ & $\begin{array}{l}\text { Mancanza di riservatezza } \\
\text { nella gestione } \\
\text { di situazioni critiche }\end{array}$ & $\begin{array}{l}\text { Difficoltà a modificare } \\
\text { l'immagine del locale } \\
\text { in caso di giudizio negativo }\end{array}$ & Non risponde & \\
\hline 3 & $\begin{array}{l}\text { Quali sono le sue } \\
\text { perplessità per la } \\
\text { realizzazione del sistema? }\end{array}$ & $\begin{array}{l}\text { Difficoltà nel rendere } \\
\text { omogenei i criteri } \\
\text { di controllo dell'A.S. }\end{array}$ & $\begin{array}{l}\text { Difficoltà a introdurre } \\
\text { l'iniziativa ai gestori } \\
\text { dei pubblici esercizi }\end{array}$ & Tempi di realizzazione & Non risponde & \\
\hline 4 & $\begin{array}{l}\text { Quali forme di comunicazione } \\
\text { trova praticabili? }\end{array}$ & $\begin{array}{l}\text { Portale interattivo con } \\
\text { identificazione del } \\
\text { pubblico esercizio } \\
\text { e esito dei controlli }\end{array}$ & Vetrofania obbligatoria & $\begin{array}{l}\text { Esposizione dell'esito } \\
\text { solo all'interno } \\
\text { del pubblico esercizio }\end{array}$ & Non risponde & \\
\hline 5 & $\begin{array}{l}\text { La comunicazione } \\
\text { dell'esito dei controlli } \\
\text { dovrebbe riguardare }\end{array}$ & $\begin{array}{l}\text { Tutti i punti monitorati } \\
\text { e i prowvedimenti } \\
\text { amministrativi }\end{array}$ & Breve giudizio & $\begin{array}{l}\text { Un valore } \\
\text { numerico da } 1 \text { a } 5\end{array}$ & Non risponde & \\
\hline 6 & $\begin{array}{l}\text { Il sistema di grading } \\
\text { dovrebbe essere } \\
\text { volontario e su richiesta? }\end{array}$ & Sì & No & Non risponde & & \\
\hline 7 & $\begin{array}{l}\text { È a conoscenza che alcuni } \\
\text { stati europei applicato } \\
\text { questo sistema? }\end{array}$ & Sì & No & Non risponde & & \\
\hline 8 & $\begin{array}{l}\text { La conoscenza di un sistema } \\
\text { di monitoraggio delle attività } \\
\text { da parte dell'A.S. effettuata } \\
\text { in modo omogeneo } \\
\text { e standardizzato sul territorio } \\
\text { le darebbe fiducia } \\
\text { nell'applicazione del sistema? }\end{array}$ & Sì & No & Non risponde & & \\
\hline 9 & $\begin{array}{l}\text { Quali parametri dovrebbero } \\
\text { essere oggetto di verifica? }\end{array}$ & $\begin{array}{l}\text { Modalità } \\
\text { di preparazione }\end{array}$ & Pulizia & $\begin{array}{l}\text { Formazione } \\
\text { del personale }\end{array}$ & $\begin{array}{l}\text { Procedure } \\
\text { di autocontrollo }\end{array}$ & $\begin{array}{l}\text { Analisi di } \\
\text { laboratorio }\end{array}$ \\
\hline 10 & $\begin{array}{l}\text { Parametri diversi } \\
\text { da quelli igienico-sanitari } \\
\text { dovrebbero essere } \\
\text { considerati indicatori? }\end{array}$ & Sì & No & Non risponde & & \\
\hline
\end{tabular}


la lettera $\mathrm{N}$ in entrambi gli ambiti ATECO il livello di copertura sia del 5,82\%. Lo svolgimento dell'attività ispettiva è regolamentato da emendamenti emanati dalla Regione Lombardia allo scopo di uniformare le prestazioni nell'evoluzione di un sistema di controlli ufficiali. In particolare il Manuale Operativo Delle Autorità Competenti Locali Aziende Sanitarie Locali Regione Lombardia Standard di Organizzazione e Funzionamento ai sensi del Reg. (CE) n. 882/2004 (Commissione Europea, 2004) in vigore dall'1 gennaio 2011, oggi in revisione 02, d'ora in poi definito Manuale ha lo scopo principale di garantire la realizzazione di un sistema di controllo basato su procedure standardizzate e nel quale ciascun livello sia sottoposto a valutazione e verifica delle modalità operative.

È evidente come l'equivalenza dei controlli regionali nonché la capacità di verificare l'operato, in termini di organizzazione e di attività e con riferimento agli standard di efficacia del sistema europeo sui controlli ufficiali mediante l'attuazione di audit interni ed esterni, di livello regionale e aziendale, sia alla base di un sistema atto a garantire sia il comparto produttivo sia il consumatore.

Nella redazione del Manuale grande attenzione è posta a garantire la realizzazione di un sistema di controllo basato su procedure stan- dardizzate e nel quale ciascun livello sia sottoposto a valutazione e verifica delle modalità operative; in concreto occorre stabilire standard minimi dei servizi ASL che svolgono attività di controllo ufficiale in campo alimentare come garanzia: i) di trasparenza nei confronti degli operatori della filiera alimentare assoggettati ai controlli e dei consumatori; ii) di omogenea valutazione delle capacità degli operatori del settore di garantire con l'autocontrollo che tutte le fasi della produzione, della trasformazione e della distribuzione di alimenti, soddisfino i requisiti di igiene fissati dai regolamenti, compresa l'identificazione e la rintracciabilità degli stessi posti in commercio;

Tabella 2. Categorizzazione del rischio di attività di ristorazione (ATECO 553).

\begin{tabular}{|c|c|c|c|c|c|}
\hline A.S.L. & $\begin{array}{l}\text { Livello di rischio } \\
\text { regionale }\end{array}$ & $\begin{array}{l}\text { Livello di rischio } \\
\text { assegnato }\end{array}$ & $\begin{array}{l}\text { Attività di } \\
\text { ristorazione (n) }\end{array}$ & $\begin{array}{c}\text { Controlli } \\
\text { dichiarati (n) }\end{array}$ & $\begin{array}{c}\text { Livello di } \\
\text { copertura (\%) }\end{array}$ \\
\hline A & 3 & da 2 a 4 & 2147 & 464 & 21,61 \\
\hline B & 3 & 4 & 2733 & 674 & 24,66 \\
\hline $\mathrm{C}$ & 3 & 3 & 1457 & 322 & 22,1 \\
\hline D & 3 & 3 & 838 & 150 & 17,9 \\
\hline $\mathrm{E}$ & 3 & 3 & 644 & 76 & 11,8 \\
\hline $\mathrm{F}$ & 3 & 2 & 366 & 180 & 49,18 \\
\hline G & 3 & 3 & 773 & 190 & 24,58 \\
\hline $\mathrm{H}$ & 3 & 3 & 3105 & 780 & 25,12 \\
\hline I & 3 & 3 & 1179 & 131 & 11,11 \\
\hline L & 3 & 2 & 1143 & 83 & 7,26 \\
\hline M & 3 & $1-2$ e 4 & 571 & 220 & 38,53 \\
\hline $\mathrm{N}$ & 3 & 1 e 3 & 1977 & 115 & 5,82 \\
\hline 0 & 3 & 3 & 600 & 149 & 24,83 \\
\hline $\mathrm{P}$ & 3 & 4 & 1881 & 500 & 26,58 \\
\hline$\underline{Q}$ & 3 & 3 & 272 & 77 & 28,31 \\
\hline
\end{tabular}

Tabella 3. Categorizzazione del rischio di pubblici esercizi (ATECO 554).

\begin{tabular}{|c|c|c|c|c|c|}
\hline A.S.L. & $\begin{array}{l}\text { Livello di rischio } \\
\text { regionale }\end{array}$ & $\begin{array}{l}\text { Livello di rischio } \\
\text { assegnato }\end{array}$ & $\begin{array}{c}\text { Pubblici } \\
\text { esercizi (n) }\end{array}$ & $\begin{array}{c}\text { Controlli } \\
\text { dichiarati (n) }\end{array}$ & $\begin{array}{l}\text { Livello di } \\
\text { copertura (\%) }\end{array}$ \\
\hline A & 4 & 4 & 3028 & 624 & 20,61 \\
\hline B & 4 & 4 & 4174 & 1087 & 26,04 \\
\hline $\mathrm{C}$ & 4 & 4 & 3379 & 311 & 9,2 \\
\hline D & 4 & 3 e 4 & 1023 & 138 & 13,49 \\
\hline E & 4 & 4 & 979 & 80 & 8,17 \\
\hline $\mathrm{F}$ & 4 & 3 & 536 & 170 & 31,72 \\
\hline G & 4 & 4 & 1400 & 193 & 13,79 \\
\hline $\mathrm{H}$ & 4 & 4 & 5913 & 1600 & 27,06 \\
\hline I & 4 & 4 & 2312 & 136 & 5,88 \\
\hline $\mathrm{L}$ & 4 & 3 & 1501 & 123 & 8,19 \\
\hline M & 4 & 4 & 1090 & 187 & 17,16 \\
\hline $\mathrm{N}$ & 4 & 1 e 4 & 1977 & 115 & 5,82 \\
\hline 0 & 4 & 4 & 719 & 168 & 23,37 \\
\hline$\underline{P}$ & 4 & 4 & 2698 & 629 & 23,31 \\
\hline Q & 4 & 4 & 545 & 120 & 22,02 \\
\hline
\end{tabular}


iii) di equivalenza dei controlli regionali alle garanzie internazionali richieste da Paesi Terzi come requisito per l'esportazione dei prodotti agroalimentari; iv) di capacità di verificare il proprio operato, in termini di organizzazione e di attività e con riferimento agli standard di efficacia del sistema europeo sui controlli ufficiali mediante l'attuazione di audit interni ed esterni (di livello regionale e aziendale).

Gli standard che le ASL devono adottare per l'organizzazione dei controlli ufficiali sono descritti nelle procedure operative delle attività che compongono il controllo ufficiale e cioè: riconoscere un sistema di categorizzazione del rischio correlato con le caratteristiche delle attività produttive, degli alimenti, dei mangimi, nonché con lo stato sanitario degli animali destinati al consumo umano; progettare un sistema di auditing (dei sistemi organizzativi e gestionali, dei processi, dei prodotti), che agisca nell'ottica del miglioramento continuo delle attività di controllo; revisionare il sistema di raccolta, elaborazione e valutazione dei dati del controllo ufficiale tenendo conto dei sistemi informatici già in essere 0 in via di realizzazione; sviluppare competenze sui controlli ufficiali nel campo della sicurezza alimentare, salute e benessere animale, secondo criteri di gestione e di assicurazione della qualità, in relazione al regolamento (CE) n. 882/2004 (Commissione Europea, 2004). Il Manuale è composto da procedure di sistema, che definiscono i criteri di carattere generale per lo svolgimento dell'attività di controllo ufficiale, e procedure speciali, che definiscono modelli operativi per la conduzione dei controlli ufficiali.

Inoltre si è evidenziato che, tra gli obiettivi indicati nella circolare n. 32/SAN/2007 (Regione Lombardia, 2007a) vi era la realizzazione del Sistema informativo della prevenzio- ne. Nel 2008 è stato creato il sistema informativo I.M.Pre.S@ (Informatizzazione Monitoraggio Prevenzione Sanitaria) definibile come uno degli strumenti fondamentali per dare concreta attuazione alla programmazione, effettuazione e valutazione degli interventi di controllo nel campo della prevenzione nei servizi medici e veterinari. Questo sistema, di fatto, nato per rispondere alle esigenze di programmazione delle attività, conformemente alle richieste della Comunità Europea, è stato realizzato con lo scopo primario di avere uno strumento di condivisione tra operatori dei diversi Servizi delle ASL e di altri Enti (ARPA, Direzione Regionale e Provinciale del Lavoro, INAIL, INPS, Comuni e Province, Organismi Paritetici) che operano sul territorio regionale in ambito preventivo, al fine di ottenere un'anagrafe delle strutture soggette all'attività di vigilanza (imprese e aziende, strutture sanitarie e socio-sanitarie, scuole, cantieri, ecc.)

Tabella 4. Domande e risposte degli esercenti dei pubblici esercizi.

\begin{tabular}{|c|c|c|c|c|c|c|}
\hline & Domanda & & & Risposte & & \\
\hline 1 & $\begin{array}{l}\text { Come giudica questa } \\
\text { iniziativa in termini } \\
\text { di ricaduta sul settore? }\end{array}$ & $\begin{array}{l}\text { Un danno assoluto } \\
(6) \\
(28)\end{array}$ & $\begin{array}{l}\text { Una opportunità } \\
\text { e incentivo } \\
\text { alla qualificazione } \\
\text { criteri di qualità (39) }\end{array}$ & $\begin{array}{l}\text { Un sistema } \\
\text { premiante } \\
\text { chi opera secondo } \\
\text { realizzazione (27) }\end{array}$ & $\begin{array}{l}\text { Una eventualità } \\
\text { perauspicabile } \\
\text { ma di difficile }\end{array}$ & - \\
\hline 2 & $\begin{array}{l}\text { Nell'eventualità lei giudichi } \\
\text { non positivamente } \\
\text { o non fattibile } \\
\text { l'iniziativa cosa le suscita } \\
\text { maggiori preoccupazioni? }\end{array}$ & $\begin{array}{l}\text { Non omogeneità } \\
\text { di controllo } \\
\text { da parte dell'A.S. } \\
\text { (44) }\end{array}$ & $\begin{array}{l}\text { Mancanza di riservatezza } \\
\text { nella gestione } \\
\text { di situazioni critiche } \\
\text { (12) }\end{array}$ & $\begin{array}{l}\text { Difficoltà a modificare } \\
\text { l'immagine del locale } \\
\text { in caso di giudizio negativo } \\
\text { (22) }\end{array}$ & $\begin{array}{l}\text { Non risponde } \\
(22)\end{array}$ & \\
\hline 3 & $\begin{array}{l}\text { Quali sono le sue } \\
\text { perplessità per la } \\
\text { realizzazione del sistema? }\end{array}$ & $\begin{array}{l}\text { Difficoltà nel rendere } \\
\text { omogenei i criteri } \\
\text { di controllo dell'A.S. (40) }\end{array}$ & $\begin{array}{l}\text { Difficoltà a introdurre } \\
\text { l'iniziativa ai gestori } \\
\text { dei pubblici esercizi (20) }\end{array}$ & $\begin{array}{l}\text { Tempi di realizzazione } \\
\text { (13) }\end{array}$ & $\begin{array}{l}\text { Non risponde } \\
\text { (27) }\end{array}$ & - \\
\hline 4 & $\begin{array}{l}\text { Quali forme di comunicazione } \\
\text { trova praticabili? }\end{array}$ & $\begin{array}{l}\text { Portale interattivo con } \\
\text { identificazione del } \\
\text { pbblico esercizio } \\
\text { e esito dei controlli (7) }\end{array}$ & $\begin{array}{l}\text { Vetrofania obbligatoria } \\
\text { (14) }\end{array}$ & $\begin{array}{l}\text { Esposizione dell'esito } \\
\text { solo all'interno } \\
\text { del pubblico esercizio } \\
(29)\end{array}$ & $\begin{array}{l}\text { Non risponde } \\
(29)\end{array}$ & - \\
\hline 5 & $\begin{array}{l}\text { La comunicazione } \\
\text { dell'esito dei controlli } \\
\text { dovrebbe riguardare }\end{array}$ & $\begin{array}{l}\text { Tutti i punti monitorati } \\
\text { e i provvedimenti } \\
\text { amministrativi }(31)\end{array}$ & $\begin{array}{l}\text { Breve giudizio } \\
\text { (19) }\end{array}$ & $\begin{array}{l}\text { Un valore } \\
\text { numerico da } 1 \text { a } 5 \\
(31)\end{array}$ & $\begin{array}{l}\text { Non risponde } \\
\text { (19) }\end{array}$ & - \\
\hline 6 & $\begin{array}{l}\text { Il sistema di grading } \\
\text { dovrebbe essere } \\
\text { volontario e su richiesta? }\end{array}$ & Sì (27) & No (73) & - & - & - \\
\hline 7 & $\begin{array}{l}\text { È a conoscenza che alcuni } \\
\text { stati europei applicato } \\
\text { questo sistema? }\end{array}$ & Sì (14) & No (86) & - & - & - \\
\hline 8 & $\begin{array}{l}\text { La conoscenza di un sistema } \\
\text { di monitoraggio delle attività } \\
\text { da parte dell'A.S. effettuata } \\
\text { in modo omogeneo } \\
\text { e standardizzato sul territorio } \\
\text { le darebbe fiducia } \\
\text { nell'applicazione del sistema? }\end{array}$ & Sì (43) & No (57) & - & - & - \\
\hline 9 & $\begin{array}{l}\text { Quali parametri dovrebbero } \\
\text { essere oggetto di verifica? }\end{array}$ & $\begin{array}{l}\text { Modalità } \\
\text { di preparazione(16) }\end{array}$ & $\begin{array}{l}\text { Pulizia } \\
\text { (16) }\end{array}$ & $\begin{array}{l}\text { Formazione } \\
\text { del personale (11) }\end{array}$ & $\begin{array}{l}\text { Procedure } \\
\text { di autocontrollo (39) }\end{array}$ & $\begin{array}{c}\text { Analisi di } \\
\text { laboratorio (18) }\end{array}$ \\
\hline 10 & $\begin{array}{l}\text { Parametri diversi } \\
\text { da quelli igienico-sanitari } \\
\text { dovrebbero essere } \\
\text { considerati indicatori? }\end{array}$ & Sì (36) & No $(36)$ & Non risponde (28) & - & - \\
\hline
\end{tabular}


contenente la tipologia dei controlli: sopralluoghi, verifiche documentali, inchieste per infortuni sul lavoro, campionamenti ecc., effettuati dagli stessi operatori. Non da ultimo l'obiettivo secondario era quello di avere la disponibilità di estrarre report e statistiche periodiche sulle imprese/strutture presenti nel territorio regionale e sulle attività di controllo svolte, in realizzazione al fondamento d trasparenza dell'attività della Pubblica Amministrazione, e in coerenza alla creazione di un data base nel quale le informazioni presenti in I.M.Pre.S@ devono poter essere rese disponibili, a oggi, ai soggetti che hanno titolo e ruolo nell'attività di controllo.

La standardizzazione delle informazioni, normalmente operata tramite una codifica condivisa, determina, da una parte un difetto dovuto all'informatizzazione del dato che porta alla sintesi dei risultati senza alcun dettaglio, ma, dall'altra, è evidente il vantaggio di avere un data-base della classificazione delle informazioni comune che offre la possibilità di confrontare i dati ottenuti dai diversi Enti coinvolti. Al fine di standardizzare le informazioni da condividere nel Sistema I.M.Pre.S@ sono stati anche definiti gli elementi dell'attività di controllo giudicati essenziali a garantire un efficace scambio informativo tra Servizi ed Enti e che permettono un'adeguata descrizione e caratterizzazione del controllo. Questi elementi definiscono il tracciato record, struttura dei dati, in cui sono individuate e codificate tutte le informazioni utili (data di controllo, identificativo del soggetto controllato, denominazione del soggetto, provincia, comune, indirizzo, tipologia struttura, motivazione del controllo, tipologia del controllo e non conformità riscontrate) a descrivere in maniera sintetica, ma sufficiente, un intervento di vigilanza in una azienda/struttura.

L'implementazione del sistema I.M.Pre.S@ prevede che tutta l'attività di controllo/vigilanza, svolta dalle ASL della Regione Lombardia, sia registrata in un unico contenitore che sia in grado di restituire i dati inseriti nel formato richiesto. Questo sistema di registrazione, per il quale potrebbe essere necessario ipotizzare di apportare modifiche e ampliamenti, consentirebbe la gestione delle informazioni necessarie al modello di comunicazione del Rischio, quale per esempio in virtù di un trasferimento dei dati in un portale Regionale dove potrebbero essere pubblicati gli esiti dei controlli nella Ristorazione Pubblica con un'immediata e facile fruizione delle informazioni da parte del consumatore. Per verificare la possibilità di un ampliamento di tale modello è stato proposto un questionario a 107 gestori di Pubblici Esercizi in cui si è valutato com'era percepita la possibile introduzione di un sistema di valutazione dell'aderenza del gestore alla normativa vigente coerente alla normativa sulla sicurezza alimentare. Le risposte sono raccolte nella Tabella 4.

L'attribuzione della Categoria di Rischio effettuata dalle diverse ASL rileva ancora un andamento poco uniforme, sembra che poche ASL abbiano effettivamente effettuato una attribuzione specifica per ogni singola attività, questo vuol dire che ad oggi mediamente, un bar viene ispezionato ogni 6 anni ed un ristorante ogni 4. Questo indice di copertura è sicuramente insufficiente a sostenere un progetto d'introduzione ufficiale di valutazione mediante grading, in quanto i principi di equità, previsti anche dalle normative comunitarie, non possono essere rispettati. I dati epidemiologici d'incidenza delle malattie 0 intossicazioni trasmesse dagli alimenti, raccolti nelle pubblicazioni L'analisi della Epidemiologia delle malattie trasmesse da alimenti in Emilia-Romagna Periodo 2009-2010 (Gruppo regionale MTA, 2010) e Il Sistema di Sorveglianza dei Focolai Epidemici di Malattie Trasmesse da Alimenti della Regione Piemonte- Rapporto 2010 (Regione Piemonte, 2010) evidenziano come la Ristorazione Pubblica, dopo la casa privata, sia il luogo più rappresentato per la preparazione dell'alimento con il $53,9 \%$ verso il $41,6 \%$ rispettivamente. In particolare nella Regione Piemonte la maggior percentuale di casi di malattia nel periodo 2002-2009 è avvenuta nella ristorazione pubblica (responsabile del $39 \%)$; seguita dai casi verificatisi presso le abitazioni private (32\%) e i casi della ristorazione collettiva (24\%). La Regione EmiliaRomagna, nel 2010, riporta invece che i casi di malattia nella ristorazione pubblica sono stati il 41\%; i casi avvenuti presso abitazioni civili hanno costituito il 58\%; mentre nessun caso di malattia è stato osservato nella ristorazione collettiva. Le attività, maggiormente identificate quale origine delle Malattie Trasmesse dagli Alimenti (MTA), segnalate da entrambe le Regioni, sono in ordine progressivo: scorretto mantenimento della temperatura di conservazione dell'alimento; cottura inadeguata; consumo di cibo crudo; provenienza incerta dell'alimento; comportamenti igienici scorretti; tempo di latenza in giorni tra preparazione $\mathrm{e}$ consumo del cibo.

La nuova categorizzazione del rischio dovrebbe quindi prendere in considerazione questi aspetti e assegnare categorie di rischio appropriate alle attività di ristorazione pubblica. Applicando queste valutazioni preliminari si dovrebbe introdurre per le diverse attività della filiera dalla produzione primaria alla somministrazione, una nuova frequenza d'ispezioni così suddivisa: Classe di Rischio 4 n.1 ispezione ogni 3 anni; Classe di Rischio 3 n.1 ispezione ogni 2 anni, Classe di Rischio 2 n.1 ispezione/anno e Classe di Rischio 1 n.2 ispezioni ed 1 audit all'anno. Tra gli elementi di criticità, rispetto all'adozione del sistema, gli aspetti di natura giuridica sono quelli che richiedono un approfondimento. La realizza- zione di un Portale Regionale non può prescindere dalla valutazione minuziosa di tutti quegli elementi che potrebbero da un lato ostacolare l'iniziativa dall'altro ledere i diritti civili, quali la normativa sulla Privacy e sulla Libera Concorrenza.

La legislazione sulla privacy in Italia è attualmente contenuta nella Costituzione agli articoli 15 e 21, nel Codice penale (Capo III Sezione IV) e nel Decreto legislativo 30 giugno 2003, n. 196 (Repubblica Italiana, 2003), intitolato Codice in materia di protezione dei dati personali e noto impropriamente anche come Testo unico sulla privacy. Sull'applicazione della normativa vigila l'Autorità Garante per la protezione dei dati personali, istituita dalla $\mathrm{L}$. 675/1996 e confermata dal Testo Unico del 2003. Le finalità del D. Lgs 196/2003 consistono nel riconoscimento del diritto del singolo sui propri dati personali e, conseguentemente, nella disciplina delle diverse operazioni di gestione (tecnicamente trattamento) dei dati, riguardanti la raccolta, l'elaborazione, il raffronto, la cancellazione, la modificazione, la comunicazione o la diffusione degli stessi. Il diritto assoluto di ciascuno sui propri dati è esplicitamente riconosciuto dall'art. 1 del testo unico, in cui si afferma: Chiunque ha diritto alla protezione dei dati personali che lo riguardano. Tale diritto appartiene alla categoria dei diritti della personalità. Lo scopo della legge non è quello di impedire il trattamento dei dati, ma di evitare che questo avvenga contro la volontà dell'avente diritto, ovvero secondo modalità pregiudizievoli. Infatti, il testo unico definisce i diritti degli interessati, la modalità di raccolta e i requisiti dei dati, gli obblighi di chi raccoglie, detiene o tratta dati personali e le responsabilità e sanzioni in caso di danni. Chiaramente le Autorità competenti locali devono uniformarsi, mediante l'adozione di appositi regolamenti interni, a quanto prescritto dalla normativa vigente in tema di privacy, tuttavia le Norme in tema di privacy debbano essere lette compatibilmente con gli obblighi normativi dell'attività di controllo ufficiale.

Nel marzo del 2011 (Repubblica Italiana, 2011) sono state pubblicate le linee guida in materia di trattamento di dati personali contenuti anche in atti e documenti amministrativi, effettuato da soggetti pubblici per finalità di pubblicazione e diffusione sul web - che offrono numerosi spunti sulle possibili modalità di realizzazione del portale. Le linee guida hanno lo scopo di definire un primo quadro unitario di misure e accorgimenti finalizzati a individuare opportune cautele che i soggetti pubblici sono tenuti ad applicare in relazione alle ipotesi in cui effettuano, in attuazione alle disposizioni normative vigenti, attività di comunicazione o diffusione di dati personali sui propri siti istituzionali per finalità di trasparenza, pubblicità dell'azione amministrativa, nonché 
di consultazione di atti su iniziativa di singoli soggetti. Le amministrazioni possono inoltre pubblicare sul proprio sito web informazioni che contengono dati personali, eventualmente anche tratti da atti e documenti amministrativi, qualora tale divulgazione, sorretta da un'adeguata motivazione, costituisca un'operazione strettamente necessaria al perseguimento delle finalità assegnate all'amministrazione da specifiche leggi o regolamenti, e riguardi informazioni utili a far conoscere ai destinatari le sue attività e il suo funzionamento. Trasparenza, pubblicità e consultabilità sono le situazioni contemplate dalla norma per la realizzazione di un sito web, nella fattispecie il portale rientrerebbe nella casistica di trasparenza e consultabilità. Tra gli accorgimenti tecnici da utilizzare, riguardo alle finalità sicuramente importanti da perseguire sono quelli legati ai motori di ricerca e al mantenimento della diffusione dei dati. La tutela dell'iniziativa economica privata è contenuta nella Costituzione della Repubblica Italiana nella Parte prima dedicata ai diritti e doveri dei cittadini che definisce, all'art.41, i rapporti economici: L'iniziativa economica privata è libera. Non può svolgersi in contrasto con l'utilità sociale o in modo da recare danno alla sicurez$z a$, alla libertà, alla dignità umana. La legge determina i programmi e $i$ controlli opportuni perché l'attività economica pubblica e privata possa essere indirizzata e coordinata a fini sociali. Nella Carta dei diritti fondamentali dell'Unione Europea (2000/C 364/01; Commissione Europea, 2000a) articolo 16 inerente la Libertà d'impresa è espressamente scritto: È riconosciuta la libertà d'impresa, conformemente al diritto comunitario e alle legislazioni e prassi nazionali.

La tutela del libero mercato e della concorrenza è disciplinata dalla legislazione italiana sia con la Legge 10 ottobre 1990, n. 287 (Repubblica Italiana, 1990a) - Norme per la tutela della concorrenza e del mercato-, istituita sia con l'Autorità Garante della Concorrenza e del Mercato, meglio nota come Antitrust istituita anch'essa nel 1990. Nel concetto di libertà di concorrenza è, però, opportuno distinguere due differenti piani: accanto al profilo marcatamente soggettivo-relazionale della libertà di concorrenza intesa come diritto di libertà del singolo, corrispondente ad un'ottica microeconomica, si pone, infatti, in una prospettiva macroeconomica, il profilo oggettivo strutturale del libero gioco della concorrenza intesa come struttura portante dell'economia di mercato, come garanzia di una certa forma e funzionalità del mercato. La dottrina, nel tentativo di chiarire il senso e la portata dell'inerenza della libera concorrenza all'iniziativa economica, si è, però, aspramente divisa, nell'individuarne gli specifici valori di riferimento, tra il momento garantistico del riconoscimento dell'iniziativa economica come diritto di libertà, da un lato, ed il momento autoritativo dell'imposizione di limiti dettati da esigenze di interesse generale, dall'altro. La Legge 689/81 (Repubblica Italiana, 1981) sulle modifiche del sistema penale definisce i diritti dell'interessato, infatti l'articolo 18 stabilisce che Entro il termine di trenta giorni dalla data della contestazione o notificazione della violazione, gli interessati possono far pervenire all'autorità competente a ricevere il rapporto a norma dell'articolo 17 scritti difensivi e documenti e possono chiedere di essere sentiti dalla medesima autorità. Il diritto dell'interessato non è limitato né in rapporto alla sanzione né riguardo al tipo di procedimento. La pubblicazione di un giudizio negativo potrebbe configurarsi come violazione alle disposizioni della norma e sarebbe inesistente perché reso senza attendere il deposito di un ricorso amministrativo, in violazione dell'articolo 7 della legge 241/90 (Repubblica Italiana, 1990b)

\section{Conclusioni}

La comunicazione del rischio è un elemento chiave per assicurare che i consumatori siano informati sugli eventuali rischi identificati e per evitare che insorgano preoccupazioni infondate sulla sicurezza degli alimenti. La comunicazione rappresenta uno strumento indispensabile per orientare la fiducia del cittadino verso le Autorità che si occupano di sicurezza alimentare, in modo che $i$ cittadini e le parti interessate ricevano informazioni rapide, affidabili, obiettive e comprensibili nei settori di sua competenza (Reg CE 178 del 2002; Commissione Europea, 2002).

La gamma di attività economiche che, dopo la legge regionale 30/03 (Regione Lombardia, 2003) rientra nel governo dei pubblici esercizi con preparazione e somministrazione di alimenti, si è fatta quanto mai variegata con un decadimento della professionalità degli operatori del settore. Gli operatori sono però pronti ad accettare un sistema che, se da un lato sembra penalizzarli, dall'altro può essere di stimolo e di riconoscimento per lavorare con professionalità e qualità. Questo lavoro, preliminare alla redazione di uno schema di valutazione con attribuzione di un punteggio, con l'adozione di sistemi fruibili dal consumatore quali vetrofanie 0 di un portale dedicato, quale nuova modalità di valutazione dei pubblici esercizi, ha permesso di valutare da un lato la sostenibilità normativa, mentre dall'altro evidenzia una difficoltà oggettiva nell'implementare il numero delle ispezioni necessarie legate a problemi di organico dei Dipartimenti della ASL.

\section{Bibliografia}

Boehnke RH, 2000. International survey on public posting of restaurant inspection reports, and/or grade card posting schemes based upon health inspections. Region of Ottawa-Carleton Health Department ed., Ottawa, Canada.

Commissione Europea, 2000a. Carta dei diritti fondamentali dell'Unione Europea. Disponibile al sito: www.europarl. europa.eu/charter/pdf/text_it.pdf

Commissione Europea, 2000b. Libro bianco sulla sicurezza alimentare 12.1.2000 COM(1999) 719 definitivo. Disponibile al sito: ec.europa.eu/dgs/ health_consumer/library/pub/pub06_it.pdf

Commissione Europea, 2002. Regolamento del Parlamento europeo e del Consiglio del 28 gennaio 2002 che stabilisce i principi e i requisiti generali della legislazione alimentare, istituisce l'Autorità europea per la sicurezza alimentare e fissa procedure nel campo della sicurezza alimentare, 178/2002/CE. In: Gazzetta Ufficiale, L 31/1, 01-02-2002.

Commissione Europea, 2004. Regolamento del Parlamento europeo e del Consiglio del 29 aprile 2004 relativo ai controlli ufficiali intesi a verificare la conformità alla normativa in materia di mangimi e di alimenti e alle norme sulla salute e sul benessere degli animali, 882/2004/CE. In: Gazzetta Ufficiale, L 165/1, 30-04-15.

Gruppo regionale MTA, 2010. Epidemiologia delle malattie trasmesse da alimenti in Emilia-Romagna aggiornamento 2010. Regione Emilia-Romagna, Assessorato Politiche per la Salute ed., Bologna, Italy.

McDean HC, 1997. Dairy products. In: Whitten D0, Whitten BE, eds. Handbook of American business history: extractives, manufacturing, and service. Greenwood Publishing Group, Westport, CT, USA, pp 141-160.

Regione Lombardia, 2003. Disciplina delle attività di somministrazione di alimenti e bevande. Legge 24 dicembre 2003, n. 30 . Disponibile al sito: www.tuttocamere.it/ files/regione/LOMBARDIA_2003_30.pdf

Regione Lombardia, 2007a. Indicazioni operative per la predisposizione e presentazione dei Piani Integrati delle attività di prevenzione e dei controlli ex DDGR 4799/2007. Circolare Regionale Direzione Generale Sanità n. 32/SAN del 18 ottobre 2007. Disponibile al sito: www.icps.it/fitoweb290/07_32san.pdf

Regione Lombardia, 2007b. Programmazione e coordinamento degli interventi in materia di controllo ufficiale dei prodotti alimentari in Lombardia. Circolare Direzione Generale Sanità n. 6/SAN/2007. 
Disponibile al sito: normativasan.servizirl.it/port/GetNormativaFile?fileName $=79$ 4_07_06san.pdf

Regione Piemonte, 2010. Il sistema di sorveglianza dei focolai epidemici di malattie trasmesse da alimenti della regione Piemonte. Centro di Riferimento Regionale per la Sorveglianza, la Prevenzione e il Controllo delle Malattie Trasmesse da Alimenti ed., Torino, Italia.

Repubblica Italiana, 1981. Modifiche al sistema penale. Legge 24 novembre 1981 n 689 , 30 novembre 1981 n 329 . Disponibile al sito: http://www.comune.jesi.an.it/ MV/leggi/689-81.htm

Repubblica Italiana, 1990a. Norme sulle intese sull'abuso di posizione dominante e sulle operazioni di concentrazione. Legge 10 ottobre n 287, 13 ottobre 1990, n 240. Disponibile al sito: http:/www.agcm.it/normativa/concorrenza/4531-legge-10-ottobre-1990-n-287-norme-per-la-tutela-dellaconcorrenza-e-del-mercato.html

Repubblica Italiana, 1990b. Nuove norme in materia di procedimento amministrativo e di diritto di accesso ai documenti amministrativi. Legge 7 agosto n 241, 18 agosto 1990. Disponibile al sito: archivio.digitpa.gov.it/sites/default/files/no rmativa/Legge_241_1990.pdf

Repubblica Italiana, 1995. Atto di indirizzo e coordinamento alle regioni e province autonome sui criteri, D.P.R. 14 luglio 1995, 7 novembre 1995 n 260. Disponibile al sito: faolex.fao.org/docs/texts/ita9945.doc

Repubblica Italiana, 2003. Codice in materia di protezione dei dati personali. Decreto Legislativo 30 giugno 2003 n. 196, 29 luglio $2003 \mathrm{n}$ 123. Disponibile al sito: http://www.privacy.it/codiceprivacy.html

Repubblica Italiana, 2011. Linee guida in materia di trattamento di dati personali contenuti anche in atti e documenti amministrativi, effettuato da soggetti pubblici per finalità di pubblicazione e diffusione sul web, 2 marzo 2011 n. 64 . Disponibile al sito: http://www.garanteprivacy.it/web/guest/home/docweb/-/docwebdisplay/docweb/1793203 